\title{
CPT Violation: What and where to look for
}

\author{
Nick E. Mavromatos \\ King's College London, Department of Physics, London WC2R 2LS, U.K.
}

\begin{abstract}
In this review I classify the possible ways of CPT violation, and I describe briefly their phenomenology, in both terrestrial and astrophysical experiments, including antimatter factories, neutral mesons and neutrinos, and discuss the various sensitivities. I also pay attention to disentangling genuine quantum-gravity induced CPT violation from 'fake' violation due to ordinary matter effects. A particularly interesting situation arises when the breaking of CPT invariance is through unitarity violations, in the sense of the matter theory being viewed as an effective field theory, entangled with decoherening quantum gravity "environments". In such a case the quantum mechanical CPT operator is ill defined due to another mathematical theorem, and one has novel effects associated with CPT Violating modifications of Einstein-Podolsky-Rosen type correlations of entangled meson states in B and $\phi$ meson factories.
\end{abstract}

Keywords: CPT, Quantum Gravity, Decoherence, Phenomenology

PACS: 11.30.Er 04.70.Dy 03.65.Ud 03.65.Yz 13.25.Es 14.60.Pq

\section{INTRODUCTION: CPT THEOREM}

A century after Einstein's annus mirabilis, and ninety years after his revolutionary proposal on the dynamical nature of space time, which is the basis for the classical theory of General Relativity, we are still lacking a consistent theory that would describe the quantum nature of space time at short-distance scales, of order of the Planck length $10^{-35}$ $\mathrm{m}$. Any complete theory of quantum gravity is bound to address fundamental issues, directly related to the emergence of space-time and its structure at energies beyond the Planck energy scale $M_{P} \sim 10^{19} \mathrm{GeV}$. From our relatively low energy experience so far, we are lead to expect that a theory of quantum gravity should respect most of the fundamental symmetries of particle physics, that govern the standard model of electroweak and strong interactions: Lorentz symmetry and CPT invariance, that is invariance under the combined action of Charge Conjugation (C), Parity (reflection P) and Time Reversal Symmetry (T). Actually the latter invariance is a theorem of any local quantum field theory that we can use to describe the standard phenomenology of particle physics to date. The CPT theorem can be stated as follows [1]: Any quantum theory, formulated on flat space time is symmetric under the combined action of CPT transformations, provided the theory respects (i) Locality, (ii) Unitarity (i.e. conservation of probability) and (iii) Lorentz invariance.

\section{POTENTIAL CPT VIOLATION}

If such a theorem exists, then why do we have to bother to test CPT invariance, given that all our phenomenology up to now has been based on such quantum theories ? The 
answer to this question is intimately linked with our understanding of quantum gravity.

\section{CPT Violation through Decoherence: Quantum Gravity as a 'medium', opening up the matter quantum system}

First of all, the theorem is not valid (at least in its strong form) in highly curved (singular) space times, such as black holes, or in general in space-time backgrounds of some quantum gravity theories involving the so-called quantum space-time foam backgrounds [2], that is singular quantum fluctuations of space time geometry, such as black holes etc, with event horizons of microscopic Planckian size $\left(10^{-35}\right.$ meters). Such backgrounds result in apparent violations of unitarity in the following sense: there is part of information (quantum numbers of incoming matter) "disappearing" inside the microscopic event horizons, so that an observer at asymptotic infinity will have to trace over such "trapped" degrees of freedom. This would define a "space time foam" situation [2], in which the quantum gravity ground state resembles that of a decoherening "medium" in open system quantum mechanics [3, 4, 5]. Barring the exciting possibility of holographic properties of such microscopic black holes [6], one may face, under such circumstances, a situation in which an initially pure state evolves in time to get mixed: the asymptotic states are described by density matrices, defined as follows: $\rho_{\text {out }}=\operatorname{Tr}_{M}|\psi><\psi|$, where the trace is over trapped (unobserved) quantum states, that disappeared inside the microscopic event horizons in the foam. Such a nonunitary evolution results in the impossibility of defining a standard quantum-mechanical scattering matrix, connecting asymptotic states in a scattering process: $\mid$ out $>=S \mid$ in $\rangle$, $S=e^{i H\left(t_{i}-t_{f}\right)}$, where $t_{i}-t_{f}$ is the duration of the scattering (assumed much longer than other time scales in the problem). Instead, in foamy situations, one can define an operator that connects asymptotic density matrices [7]: $\rho_{\text {out }} \equiv \operatorname{Tr}_{M} \mid$ out $><$ out $\mid=\$ \rho_{\text {in }}, \quad \$ \neq$ $S S^{\dagger}$, where the lack of factorization is attributed to the apparent loss of unitarity of the effective low-energy theory, defined as the part of the theory accessible to low-energy observers who perform scattering experiments. This defines what we mean by particle phenomenology in such situations. The \$ matrix is not invertible, and this reflects the effective unitarity loss. It is this property, actually, that leads to a violation of CPT invariance, at least in its strong form, in the sense that the generator of CPT symmetry is ill-defined. This has been shown rigorously in ref. [8]. However, as cautiously pointed out there, despite the strong violation of CPT in such a situation, one cannot exclude the possibility that a weak form of CPT invariance remains, which is reflected in the possibility of the "experimentalist" to prepare pure initial quantum states, $|\psi\rangle$, that evolve into pure asymptotic states, $|\phi\rangle$ (defining, in some sense, a "decoherence-free subspace" in the language of open systems), in such a way that CPT is preserved in the respective probabilities: $P(\psi \rightarrow \phi)=P\left(\theta^{-1} \phi \rightarrow \theta \psi\right)$, where $\theta: \mathscr{H}_{\text {in }} \rightarrow \mathscr{H}_{\text {out }}$, with $\mathscr{H}$ denoting the appropriate Hilbert state spaces. The notation is such that the CPT operator $\Theta$ acting on density matrices is: $\Theta \rho=\theta \rho \theta^{\dagger}, \quad \theta^{\dagger}=-\theta^{-1}$ (anti - unitary).

As we shall discuss in this article, such issues can, in principle, be resolved experimentally, provided of course the sensitivity of the experiments is appropriate to the order expected from theoretical models of quantum gravity. 


\section{Cosmological CPT Violation?}

Another type of possible violation of CPT [5], which falls within the remit of the theorem of [8], may be associated with the recent experimental evidence from supernovae and temperature fluctuations of the cosmic microwave background on a current era acceleration of the Universe, and the fact that more than $70 \%$ of its energy budget consists of Dark Energy. If this energy substance is a positive cosmological constant (de Sitter Universe), $\Lambda>0$, then there is a cosmic horizon, in the sense that in a flat Universe, as the data seem to indicate we live in, light emitted at some future moment $t_{0}$ in the cosmic time takes an infinite time to cover the finite horizon radius. The presence of a cosmic future horizon implies impossibility of defining proper asymptotic states, and in particular quantum decoherence of a matter quantum field theory in this de Sitter geometry, as a result of an environment of modes crossing the horizon. In view of [8], then, this would imply an analogous situation with the foam, discussed above, i.e. a strong form of CPT violation. In this particular case, as the horizon is macroscopic, one would definitely have the evolution of an initial pure quantum state to a mixed one, and probably no weak form of CPT invariance would exist, in contrast to the black-hole case [5].

\section{CPT Violation in the Hamiltonian, consistent with closed system quantum mechanics}

Another reason for CPT violation (CPTV) in quantum gravity is spontaneous breaking of Lorentz symmetry (LV), without necessarily implying decoherence. This has also been argued to occur in string theory and other models of quantum gravity [9, 10], but, in my opinion, no concrete microscopic model has been given as yet. In string theory, for instance, where a LV vacuum has been argued to exist [9], it was not demonstrated that this vacuum is the energetically preferred one. So far, most of the literature in this respect is concentrating on consistent parameterizations of extension of the standard model (SME) [9], which can be used to bound the relevant Lorentz and/or CPT violating parameters. An important difference of this approach, as compared with the decoherence one is the fact that in SME or other spontaneous Lorentz symmetry breaking approaches, the CPT Violation is linked merely with non-commutativity of the CPT operator, which otherwise is a well-defined quantum mechanical operator, with the Hamiltonian of the system. In such models there is a well defined scattering matrix, and the usual phenomenology applies. As we shall argue below, experimentally one can in principle disentangle these two types of violation, by means of appropriate observables and their time evolution properties.

\section{Order of Magnitude Estimates of Quantum Gravity Effects}

At first sight, the CPT violating effects can be estimated to be strongly suppressed. Indeed, naively, Quantum Gravity (QG) has a dimensionful coupling constant: $G_{N} \sim$ $1 / M_{P}^{2}$, where $M_{P}=10^{19} \mathrm{GeV}$ is the Planck scale. Hence, CPT violating and decoheren- 
ing effects may be expected to be suppressed by terms (with dimensions of energy) of order $E^{3} / M_{P}^{2}$, where $E$ is a typical energy scale of the low-energy probe. If such is the case, the current facilities seem far from reaching this sensitivities. But, as we shall mention below, high energy cosmic neutrino observations may well reach such sensitivities in the future. However, there may be cases where loop resummation and other effects in theoretical models may result in much larger CPT-violating effects of order:

$\frac{E^{2}}{M_{P}}$. This happens, for instance, in some loop gravity approaches to Quantum Gravity, or some non-equilibrium stringy models of space-time foam involving open string excitations [5]. Such large effects can lie within the sensitivities of current or immediate future experimental facilities (terrestrial and astrophysical), and hence can be, or are already, excluded [11].

\section{PHENOMENOLOGY}

The phenomenology of CPT Violation is complicated, as there is no single figure of merit, and the relevant sensitivities are highly model dependent. Below I outline briefly, the main phenomenological searches for CPT violation, and the respective sensitivities, in the various major approaches to CPT Violation outlined above.For further details see refs. [9, 10, 5, 11].

\section{CPT Violation in the Hamiltonian}

The main activities in this area concern: (i) Lorentz violation in extensions of the standard model [9, 10], which provide an exhaustive phenomenological study of various Lorentz and CPT Violating effects in a plethora of atomic, nuclear and particle (neutrinos) physics experiments. Modified Dirac equation in the presence of external gauge fields can be used for constraining CPTV and Lorentz violating parameters by means of, say, antimatter factories [12]. I will not discuss further such tests here. For details I refer the reader to the literature [9, 10, 13]. Specific tests for CPTV in the antihydrogen system, via precision measurements of its hyperfine structure, can be found in [14]. At present, the most sensitive of the parameters of CPT and Lorentz violation, $b$ in the notation of [10] can be constrained to be smaller than $b<10^{-27} \mathrm{GeV}$ (or $b<10^{-31} \mathrm{GeV}$ in masers). Since there is no microscopic model underlying the standard model extension at present it is difficult to interpret such small bounds, as far as sensitivity at Planckian energy scales is concerned. In the naive dimensional estimate that $[b]=E^{2} / M_{Q G}$, where $M_{Q G}$ is a quantum gravity scale, one obtains sensitivities to scales up to $M_{Q G}=10^{27}$ $\mathrm{GeV}$, thereby tending to excluding linear suppression by the Planck mass. However, if, as expected in such models, there are quadratic (or higher) suppressions by $M_{P}$, then we are some 10 orders of magnitude away from Planck scale physics at present.

(ii) Tests of modified dispersion relations for matter probes. Stringent tests for charged fermions (e.g. electrons) are provided by synchrotron radiation measurements from astrophysical sources, e.g. Crab nebula [15, 11], whilst the most accurate tests of modified dispersion relations for photons are at present provided by Gama-Ray-Burst observa- 
tions on arrival times of radiation at various energy channels [16]. The present observations of photons from Gamma Ray Bursts and Active Galactic Nuclei (AGN) concern energy scales up to a few $\mathrm{MeV}$ at most. If the quantum gravity effects are then linearly suppressed by the quantum gravity scale, then the sensitivity is $M_{Q G}>10^{16} \mathrm{GeV}$, otherwise is much less. There is an issue here concerning the universality of quantum gravity effects. As argued in [17], studies in certain microscopic models of modified dispersion relations in string theory, have indicated that only photons and standard model gauge bosons may be susceptible to quantum gravity effects in some models. This is due to some stringy gauge symmetry protection of chiral matter particles against such effects, to all orders in perturbation theory in a low-energy quantum field theory context. It is in the above sense that it is important to obtain limits on such effects from various sources and for various systems.

(iii) As a final comment in this type of CPT violation we also mention the possibility that quantum gravity may induce non hermiticity of the effective low energy Hamiltonian, in the sense of complex coupling constants appearing in low energy theories [18]. This may lead to interesting phenomena, for instance complex anomalous magnetic moments of, say, protons, from which one may place stringent constraints in such effects. Since in all microscopic models we have in our disposition so far such imaginary effects do not appear we shall not pursue this discussion further inhere. However, we stress again, we cannot exclude this possibility from appearing in future microscopic models of quantum gravity.

\section{Quantum Gravity Decoherence and CPT Violation}

\section{Neutral Mesons (Single Kaon states)}

Quantum Gravity may induce decoherence and oscillations among neutral mesons, such as kaons $K^{0} \rightarrow \bar{K}^{0}$ [3, 4]. The modified evolution equation for the respective density matrices of neutral kaon matter can be parametrized as follows [3]:

$$
\partial_{t} \rho=i[\rho, H]+\delta H \rho
$$

where $H$ is the standard Kaon Hamiltonian, and the "entanglement" matrix is given by

$$
\delta H_{\alpha \beta}=\left(\begin{array}{cccc}
0 & 0 & 0 & 0 \\
0 & 0 & 0 & 0 \\
0 & 0 & -2 \alpha & -2 \beta \\
0 & 0 & -2 \beta & -2 \gamma
\end{array}\right) \text {. }
$$

Positivity of $\rho$ requires: $\alpha, \gamma>0, \quad \alpha \gamma>\beta^{2}$. Notice that $\alpha, \beta, \gamma$ violate CPT in the sense of an induced microscopic time irreversibility of [8], as being associated with decoherence, but also they violate $\mathrm{CP}$ since they do not commute with the $\mathrm{CP}$ operator [4]: $C P=\sigma_{3} \cos \theta+\sigma_{2} \sin \theta, \quad\left[\delta \not H_{\alpha \beta}, C P\right] \neq 0$.

An important remark is now in order. We should distinguish two types of CPTV: (i) CPTV within Quantum Mechanics: $\delta M=m_{K^{0}}-m_{\bar{K}^{0}}, \delta \Gamma=\Gamma_{K^{0}}-\Gamma_{\bar{K}^{0}}$. This could be due to (spontaneous) Lorentz violation (c.f. above). 
(ii) CPTV through decoherence $\alpha, \beta, \gamma$ (entanglement with QG 'environment', leading to modified evolution for $\rho$ and $\$ \neq S S^{\dagger}$ ).

The important point is that the two types of CPTV can be disentangled experimentally [4]. Experimentally, the best available bounds of $\alpha, \beta, \gamma$ parameters to date for single neutral Kaon states come from CPLEAR measurements [19] $\alpha<4.0 \times$ $10^{-17} \mathrm{GeV},|\beta|<2.3 . \times 10^{-19} \mathrm{GeV}, \gamma<3.7 \times 10^{-21} \mathrm{GeV}$, which are not much different from theoretically expected values in the most optimistic of the scenaria, involving linear Planck-scale suppression $\alpha, \beta, \gamma=O\left(\xi \frac{E^{2}}{M_{P}}\right)$. For more details we refer the reader to the literature [4, 13].

Neutral Meson factories: entangled meson states

The above-described decoherence formalism can be used to derive the (non-unitary) evolution of the entangled products of the $\phi$ or $\Upsilon$ decays in meson factories. The requirement of complete positivity of the respective density matrices imposes further restrictions among the decoherence parameters in that case, amounting to setting $\beta=0$, $\alpha=\gamma \neq 0[20]$.

An entirely novel observable, exclusive to a breakdown of CPT Violation through decoherence, in which case the CPT operator is not well defined, pertains to observations of the modifications of Einstein-Podolsky-Rosen entangled states of neutral mesons in meson factories ( $\phi-$ or B-factories) [21]. These modifications concern the nature of the products of the decay of the neutral mesons in a factory on the two sides of the detector. For instance, for neutral kaons, if the CPT operator is a well defined operator, even if it does not commute with the Hamiltonian of the system, the products of the decay contain states $K_{L} K_{S}$ only. On the contrary, in the case of CPT breakdown through decoherence (ill defined CPT operator), one obtains in the final state, in addition to $K_{L} K_{S}$, also $K_{S} K_{S}$ and/or $K_{L} K_{L}$ states. In a similar manner, when this formalism of CPT breaking through decoherence is applied to B-systems, one observes that flavor tagging fails in B-factories as a result of such CPTV modifications [21].

\section{Neutrinos}

Similarly to neutral mesons, one could have decoherening modifications of the oscillation probabilities for neutrinos. Due to lack of space we shall not describe the details here. We refer the interested reader in the literature [5]. We only mention that at present there is no experimental evidence in neutrino physics on quantum gravity decoherence effects, only very stringent bounds exist. Although, at first sight, it appears that neutrino anomalies, such as the LSND result [22] indicating asymmetric rates for antineutrino oscillations as compared to neutrino ones, can be fit in the context of a decoherence model with mixed energy dependences, $E$ and $1 / E$, and with different orders of the decoherence parameters between particle and antiparticle sectors [23], nevertheless recent spectral distortions from KamLand [24] indicate that standard oscillations are capable of explaining these distortions, excluding the order of decoherence in the antineutrino sector necessary to match the LSND result with the rest of the available neutrino data. This leaves us with the following bounds as far as decoherence coefficients $\gamma$ are concerned for neutrinos [25]: in a parametrization $\gamma \sim \gamma_{0}\left(\frac{E}{\mathrm{GeV}}\right)^{n}$, with $n=0,2,-1$, we have: (a) $n=0, \gamma_{0}<3.5 \times 10^{-23} \mathrm{GeV}$, (b) $n=2, \gamma_{0}<0.9 \times 10^{-27} \mathrm{GeV}$ (compare with the neutral Kaon case above), and (c) $n=-1, \gamma_{0}<2 \times 10^{-21} \mathrm{GeV}$. Very stringent limits on quantum gravity decoherence may be placed from future observations of high-energy neutrinos 
from extragalactic sources [5] (Supernovae, AGN), if, for instance, Quantum Gravity induces lepton number violation and/or flavor oscillations. We therefore see that neutrinos provide the most sensitive probe at present for tests of quantum gravity decoherence effects, provided the latter pertain to these particles.

Disentangling Matter Effects from Genuine Quantum Gravity induced Decoherence effects

However, there is an important aspect in all such decoherence searches, which should not be overlooked. This concerns "fake" decoherence effects as a result of the passage of the particle probe, e.g. neutrino, through matter media. Due to the apparent ("extrinsic") breaking of CPT in such a case, one obtains fake violations of the symmetry, which have nothing to do with genuine microscopic gravity effects. Matter effects, for instance, include standard CPTV differences in neutrino oscillation probabilities of the form:

$P_{v_{\alpha} \rightarrow v_{\beta}} \neq P_{\bar{v}_{\beta} \rightarrow \bar{v}_{\alpha}}$, non-zero result for the so-called $A_{C P T}^{r}$ asymmetry to leading order, in the case of Kaons through a regenerator [4], as well as uncertainties in the energy and oscillation length of neutrino beams, which result in damping factors in front of terms in the respective oscillation probabilities. Such factors are of similar nature to those induced by decoherence $\gamma$ coefficients [26]. Nevertheless, the energy and oscillation length dependence of the effects is different [5, 23], and allows disentanglement from genuine effects, if the latter are present and are of comparable order to the matter effects. Namely, quantum gravity effects are in general expected to increase with the energy of the neutrino probe, as a result of the fact that the higher the energy the stronger the back reaction onto the space time. This should be contrasted with ordinary matter effects, which are expected to decrease with the energy of the matter probe. Also, the damping factors in the case of neutrinos with uncertainty in their energy have a different oscillation length dependence, as compared with quantum gravity decoherence effects. Note that matter decoherence effects may be tiny [26], with the appropriate coefficients of order smaller than $\gamma_{\text {fake }}<10^{-24} \mathrm{GeV}$, thereby leading to the temptation of identifying (incorrectly) such effects with genuine microscopic effects.

\section{CONCLUSIONS}

There are various ways by means of which Quantum Gravity (QG)-induced CPT breaking can occur, which are in principle independent of each other. For instance, quantum decoherence and Lorentz Violation are in general independent effects. There is no single figure of merit of CPT violation, and the associated sensitivity depends on the way CPT is broken, and on the relevant observable. The pertinent phenomenology is not simple.

Neutrino (astro)physics may provide some of the most stringent (to date) constraints on QG CPT Violation [27]. There are interesting theoretical issues on Quantum Gravity decoherence and neutrinos, which go as far as the origin of neutrino mass differences (which could even be due to space time foam effects, thus explaining their smallness), and their contributions to the Dark Energy [28].

Quantum-Gravity induced decoherence CPTV, in which the CPT generator is ill defined, may lead to interesting novel observables with high sensitivity in the near future, associated with entangled state modifications in neutral meson factories $(\mathrm{B}, \phi)$.

Thus, it seems that a century after annus mirabilis, there is still a long way to go 
before an understanding of Quantum Gravity is achieved. But the challenge is there, and we think that there may be pleasant experimental surprises in the near future. This is why experimental searches of CPT violation and other quantum gravity effects along the lines presented here are worth pursuing.

\section{ACKNOWLEDGMENTS}

The author would like to thank Prof. W. Oelert and the other organizers of the LEAP05 conference in Bonn/GSI for the invitation, and for creating such a stimulating meeting.

\section{REFERENCES}

1. G. Lüders, Ann. Phys. (NY) 2 (1957), 1.

2. see: J. A. Wheeler and K. Ford, Geons, Black Holes, And Quantum Foam: A Life In Physics, (New York, USA: Norton (1998)).

3. J. R. Ellis, J. S. Hagelin, D. V. Nanopoulos and M. Srednicki, Nucl. Phys. B 241 (1984) 381.

4. J. R. Ellis, J. L. Lopez, N. E. Mavromatos and D. V. Nanopoulos, Phys. Rev. D 53 (1996) 3846

5. N. E. Mavromatos, arXiv:gr-qc/0407005 Springer Lect. Notes Phys. in press, and references therein.

6. J. M. Maldacena, Adv. Theor. Math. Phys. 2, 231 (1998) [Int. J. Theor. Phys. 38, 1113 (1999)] |arXiv:hep-th/9711200|; E. Witten, Adv. Theor. Math. Phys. 2, 253 (1998) |arXiv:hep-th/9802150|; S. Hawking, in 2003, Dublin conference on Relativity, unpublished.

7. S. W. Hawking, Comm. Math. Phys. 87 (1982) 395.

8. R. Wald, Phys. Rev. D21 (1980), 2742.

9. for a review see: V.A. Kostelecky, arXiv:hep-ph/9912528 and references therein.

10. R. Bluhm, V. A. Kostelecky and N. Russell, Phys. Rev. Lett. 82 (1999) 2254; R. Bluhm, arXiv:hep-ph/0111323 V. A. Kostelecky and M. Mewes, Phys. Rev. D 70, 031902 (2004) |arXiv:hep-ph/0308300|;

11. see for instance: D. Mattingly, arXiv:gr-qc/0502097, and references therein.

12. M. Amoretti et al., Nature 419, 456 (2002); G. Gabrielse et al. [ATRAP Collaboration], Phys. Rev. Lett. 89, 233401 (2002).

13. N. E. Mavromatos, Nucl. Instrum. Meth. B 214, 1 (2004) |arXiv:hep-ph/0305215|, and references therein.

14. E. Widemann, R.S. Hayano, M. Hori and T. Yamazaki, Nucl. Instr. Meth. B214, 31 (2004), and references therein. See also: R. Hayano and E. Widemann talks in these proceedings.

15. T. Jacobson, S. Liberati and D. Mattingly, Nature 424, 1019 (2003) |arXiv:astro-ph/0212190|.

16. G. Amelino-Camelia, J. R. Ellis, N. E. Mavromatos, D. V. Nanopoulos and S. Sarkar, Nature 393, 763 (1998) |arXiv:astro-ph/9712103|; J. R. Ellis, K. Farakos, N. E. Mavromatos, V. A. Mitsou and D. V. Nanopoulos, Astrophys. J. 535, 139 (2000) |arXiv:astro-ph/9907340|; J. R. Ellis, N. E. Mavromatos, D. V. Nanopoulos and A. S. Sakharov, Astron. Astrophys. 402, 409 (2003) |arXiv:astro-ph/0210124].

17. J. R. Ellis, N. E. Mavromatos, D. V. Nanopoulos and A. S. Sakharov, Int. J. Mod. Phys. A 19, 4413 (2004) |arXiv:gr-qc/0312044|; Nature 428, 386 (2004) |arXiv:astro-ph/0309144|.

18. L. B. Okun, arXiv:hep-ph/0210052

19. R. Adler et al. [CPLEAR collaboration], Phys. Lett. B 364 (1995) 239.

20. F. Benatti and R. Floreanini, Nucl. Phys. B 511, 550 (1998) |arXiv:hep-ph/9711240|; Phys. Lett. B 468, 287 (1999) [arXiv:hep-ph/9910508|.

21. J. Bernabeu, N. E. Mavromatos and J. Papavassiliou, Phys. Rev. Lett. 92, 131601 (2004) |arXiv:hep-ph/0310180|; E. Alvarez, J. Bernabeu, N. E. Mavromatos, M. Nebot and J. Papavassiliou, Phys. Lett. B 607, 197 (2005) |arXiv:hep-ph/0410409|; J. Bernabeu, N. E. Mavromatos, J. Papavassiliou and A. Waldron-Lauda, arXiv:hep-ph/0506025

22. A. Aguilar et al. [LSND Collaboration], Phys. Rev. D 64, 112007 (2001) |arXiv:hep-ex/0104049|.

23. G. Barenboim and N. E. Mavromatos, JHEP 0501, 034 (2005) |arXiv:hep-ph/0404014|. 
24. T. Araki et al. [KamLAND Collaboration], Phys. Rev. Lett. 94, 081801 (2005) |arXiv:hep-ex/0406035|.

25. E. Lisi, A. Marrone and D. Montanino, Phys. Rev. Lett. 85, 1166 (2000) [arXiv:hep-ph/0002053]. See also: F. Benatti and R. Floreanini, JHEP 0002, 032 (2000) |arXiv:hep-ph/0002221|.

26. M. Blennow, T. Ohlsson and W. Winter, arXiv:hep-ph/0502147 and references therein.

27. L. A. Anchordoqui, H. Goldberg, M. C. Gonzalez-Garcia, F. Halzen, D. Hooper, S. Sarkar and T. J. Weiler, arXiv:hep-ph/0506168, D. Hooper, D. Morgan and E. Winstanley, Phys. Lett. B 609, 206 (2005) [arXiv:hep-ph/0410094]; arXiv:hep-ph/0506091

28. G. Barenboim and N. E. Mavromatos, Phys. Rev. D 70, 093015 (2004) [arXiv:hep-ph/0406035]. 